\title{
Possibility of molecular targeting therapy for the treatment of cancer of unknown primary origin by analysis of intracellular signaling molecules
}

\author{
SHOICHIRO OHTA ${ }^{1-3}$, YUKIKO CHO ${ }^{1}$, MASAHARU SHIBATA ${ }^{1}$, KIMIHIRO NAGAI ${ }^{1}$, \\ TATSUO IIJIMA ${ }^{1}$, HITOAKI SAITO ${ }^{1}$, HIROTAKA ASAKURA ${ }^{2}$ and HIROSHI KOJIMA ${ }^{1}$ \\ ${ }^{1}$ Ibaraki Prefectural Central Hospital, Ibaraki Prefectural Cancer Center, Ibaraki; \\ ${ }^{2}$ Saitama Medical University, Saitama, Japan
}

Received October 19, 2011; Accepted December 9, 2011

DOI: $10.3892 /$ etm.2011.417

\begin{abstract}
Recently, antibody-mediated epidermal growth factor receptor (EGFR) blockade has become a major research focus, and a number of clinical studies on this new treatment have been started in the field of clinical oncology. This retrospective study investigated the role of KRAS gene mutations and clinical features for possibilities for new therapies in patients with cancer of unknown primary (CUP). We investigated the role of KRAS, PIK3CA and BRAF gene mutations and clinical features for possibilities for new therapies in patients with CUP. Nine patients with metastases from an unknown primary tumor were included in this retrospective study. The KRAS, BRAF and PI3KCA mutational analyses were carried out by means of PCR using genomic DNA for each PCR reaction. The mutation rate in CUP for codon 12 or 13 of the KRAS gene and for PIK3CA was lower than that in colorectal cancer, while the same mutation rate for BRAF was almost the same in the two; this means that the EGFR antibodies can possibly treat CUP.
\end{abstract}

\section{Introduction}

Cancer of an unknown primary (CUP) is defined as the presence of metastatic cancer in the absence of an identifiable primary tumor site (1). CUP occurs in a heterogeneous group of patients, and subgroups with treatment-responsive diseases that may achieve long-term, disease-free survival exist. Recently, however, the treatment of certain patients with CUP has improved substantially. The identification of treatable patients within this heterogeneous group has been made possible by the development of specialized pathological techniques that aid

Correspondence to: Dr Shoichiro Ohta, ${ }^{3}$ Present address: Kan-Etsu Hospital, 145-1 Suneori, Tsurugashima, Saitama 350-2213, Japan

E-mail: d11494@hotmail.co.jp

Key words: epidermal growth factor receptor, KRAS, cancer of unknown primary in characterizing tumors, and by the recognition that several patients have tumors that respond to chemotherapy.

Recently, antibody-mediated epidermal growth factor receptor (EGFR) blockade has become a major research focus, and much clinical research on this new treatment has been initiated in the field of clinical oncology (2). This retrospective study investigated the role of KRAS gene mutations and clinical features for possibilities for new therapies in patients with CUP.

\section{Materials and methods}

Between October 2004 and December 2009, 9 patients (4 women, 5 men; median age 70.5 years, range $54-87$ ) with metastases from an unknown primary tumor were included in this retrospective study. The definition of CUP syndrome covers patients with histologically confirmed metastatic cancer, when the combination of a complete medical history, accurate physical examination and laboratory tests, and conventional diagnostic imaging procedures (i.e., chest X-ray, abdominal ultrasonograph and, on the basis of suspected primary disease, CT, magnetic resonance imaging, mammography and endoscopic procedures) have failed to identify the primary site. The histological types and sites of metastases are summarized in Table I.

The blood concentrations of CA19-9, CA15-3, CA125 and carcinoembryonic antigen (CEA), and the most commonly used markers - the leukocyte common antigen, cytokeratins, chromogranin and vimentin - were assessed in most cases for histopathological diagnosis.

DNA extraction from formalin-fixed, paraffin-embedded (FFPE) tumor tissue sections. Tumor cell-rich areas of H\&E-stained sections were marked under a microscope, and tissues were scratched from the same areas of deparaffinized unstained sections. DNA for pieces of the scratched tissue samples was isolated using the QIAamp FFPE Tissue kit (Qiagen KK, Tokyo, Japan).

Mutational analyses. KRAS, BRAF and PI3KCA mutational analyses were carried out by means of PCR using $100 \mathrm{ng}$ of genomic DNA for each PCR reaction. All PCR products were directly sequenced using an ABI Prism 377 (Applied 
Table I. Clinicopathological findings.

\begin{tabular}{|c|c|c|c|c|c|c|c|c|}
\hline Case & Gender & $\begin{array}{l}\text { Age } \\
\text { (years) }\end{array}$ & PS & $\begin{array}{l}\text { Pathological } \\
\text { diagnosis }\end{array}$ & $\begin{array}{l}\text { Positive } \\
\text { marker }\end{array}$ & Treatment & $\begin{array}{c}\text { Prognosis } \\
\text { (months) }\end{array}$ & $\begin{array}{l}\text { Tumor } \\
\text { location }\end{array}$ \\
\hline 1 & $\mathrm{~F}$ & 74 & 1 & Malignant mesethelioma & Serum CA125 & Resection & 4 & Abdomen \\
\hline 2 & M & 80 & 1 & Squamous carcinoma & ND & Resection irradiation & 3 & Skeletal \\
\hline 3 & M & 87 & 2 & Adenocarcinoma & Serum CEA & $\mathrm{BSC}$ & 1 & Pleura \\
\hline 4 & M & 79 & 3 & Undifferentiated carcinoma & ND & $\mathrm{BSC}$ & 1 & Bone marrow \\
\hline 5 & F & 78 & 1 & $\begin{array}{l}\text { Epithelial, maybe } \\
\text { adenocarcinoma }\end{array}$ & AE1/AE3 CAM5.3 & Resection & 1 & Hip joint \\
\hline 6 & $\mathrm{~F}$ & 74 & 3 & Maybe adenocarcinoma & CK7 & $\mathrm{BSC}$ & 1 & Pleura \\
\hline 7 & M & 81 & 4 & Squamous carcinoma & ND & $\mathrm{BSC}$ & 2 & Liver \\
\hline 8 & M & 54 & 1 & Neuroendocrine carcinoma & CD56 & CDDP CPT11 & 13 & Liver \\
\hline 9 & $\mathrm{~F}$ & 69 & 1 & $\begin{array}{l}\text { Adenocarcinoma } \\
\text { mucinous type }\end{array}$ & $\begin{array}{l}\text { CK7, CK20 CEA } \\
\text { CA19-9 AFP CA125 }\end{array}$ & GEM PTX TS-1 & 11 & Lymph node \\
\hline
\end{tabular}

PS, physical status; F, female; M, male; ND, not detected; BSC, best supportive care; CDDP, cisplatin; CPT11, irinotecan; GEM, gemcitabine; PTX, paclitaxel.

Table II. Sequence data.

\begin{tabular}{|c|c|c|c|c|c|c|}
\hline Case & KRAS exon 12 & KRAS exon 13 & $\begin{array}{c}\text { BRAF exon } \\
15 \mathrm{~V} 600 \mathrm{E}\end{array}$ & $\begin{array}{c}\text { PI3KCA exon } 9 \\
\text { E542K }\end{array}$ & $\begin{array}{c}\text { PI3KCA exon } 9 \\
\text { E545/D }\end{array}$ & $\begin{array}{c}\text { PI3KCA exon } 20 \\
\text { H1047R }\end{array}$ \\
\hline
\end{tabular}

\begin{tabular}{|c|c|c|c|c|c|c|}
\hline 1 & - & - & - & - & - & - \\
\hline 2 & - & - & - & - & - & - \\
\hline 3 & - & - & + & - & - & - \\
\hline 4 & - & - & - & - & - & - \\
\hline 5 & - & - & - & - & - & - \\
\hline 6 & - & - & - & - & - & - \\
\hline 7 & + & - & - & - & - & - \\
\hline 8 & - & - & - & - & - & - \\
\hline 9 & - & - & - & - & - & - \\
\hline
\end{tabular}

+ , positive; -, negative. By analyzing one representative tumor sample in each patient, KRAS mutation in codon 12 was found in one (11\%) of the CUP cases. Mutations were not found in codon 13. Of the 11 tumors, 1 (11\%) exhibited BRAF mutations at codon 600. PIK3CA mutations were not found.

Biosystems, Foster City, CA, USA) and were then evaluated by means of Sequence Navigator software (Applied Biosystems). Each sequence was carried out at least twice, starting from an independent amplification reaction.

$K R A S$. Exon 1 of the KRAS gene was amplified in order to seek potential mutations on the two foremost codons (12 and 13), which have been reported to be mutated in colorectal cancer (CRC). PCR amplification was carried out using a standard protocol and previously described primers and conditions (2).

$B R A F$. Exons 11 and 15 of the BRAF gene, including the classical mutation V600E, were amplified using previously described conditions and primers (2).

PI3KCA. Exons 9 and 20 of the $\alpha$ polypeptide (the catalytic subunit of the PI3K protein; PI3KCA), which are frequently mutated in CRC, were amplified using a standard PCR protocol and previously described primers (3).

\section{Results}

Clinicopathological findings. Six specimens were adenocarcinomas, two were squamous and one was a neuroendocrine tumor. The histological type of 1 case was not diagnosed despite the use of special immunochemistry. Three cases underwent surgery and 2 were administered chemotherapies. The median survival time was 7 months. The 2 chemotherapy cases had longer survival times, 11 and 13 months, respectively (Table I). Serum CA125 was elevated in 2 cases, and neuroendocrine marker tissue CD56 was positive in 1 case (Table I). Regarding the clinical data in our cases, it was found that in most of them, histological types could be diagnosed using light microscopy, and tissue and markers in the blood served 
as auxiliary diagnosis. Markers were particularly useful in diagnosing neuroendocrine tumors.

$K R A S$. By analyzing one representative tumor sample in each patient, KRAS mutation in codon 12 was found in $1(11 \%)$ of the CUP cases (Table II). We could not find any mutations in codon 13 .

$B R A F$. Of the 11 tumors, $1(11 \%)$ exhibited BRAF mutations at codon 600 (Table II).

PIK3CA. PIK3CA mutations were not found in any tumors (Table II).

\section{Discussion}

Patients with CUP should undergo clinical and pathological evaluation to identify the tumor, followed by appropriate therapy for those who have treatable tumors. Drugs are determined according to the histological type or location of the lesion $(4,5)$. As for antibody-based EGFR-targeted therapy, namely, cetuximab, it is either approved or currently in clinical trial for both squamous cell cancer and adenocarcinoma, and the effects are expected for many histological types of CUP. Also, since gene cluster mutation, which is an effect-prediction marker for this drug, has a significantly lower rate of incidence in CUP than in colon cancer, the effects of these molecular target drugs are greatly expected in CUP.

The KRAS gene encodes a small G protein on the EGFR pathway. Cetuximab and other EGFR inhibitors only work on tumors that are not mutated. However, approximately $50 \%$ of KRAS wild-type patients do not benefit from anti-EGFR treatment (6). To identify the possible causes for this therapy failure, we suggest including mutation analyses of all three EGFR downstream genes as predictive markers in further prospective clinical trials on EGFR-directed therapy of any kind, i.e., not only for CRC. Of note, however, it has been shown that the failure of EGFR antibody therapy may be caused by the activation of mutations of EGFR-dependent signaling molecules. Two main EGFR-dependent signaling pathways are the KRAS-BRAF and the PIK3CA-PTEN-AKT pathways, which regulate cell proliferation, survival, cell growth, apoptosis resistance, invasion and migration (7). In various clinical studies that included cetuximab or panitumumab in second- or third-line therapy, only patients with wild-type KRAS responded to therapy (up to $50 \%$ of all patients), whereas patients with tumors exhibiting KRAS mutations had a response rate between 0 and 6\% (6). Similarly, as shown for KRAS mutations, mutations of other EGFR-dependent signaling molecules, such as BRAF and PIK3CA, may confer resistance to EGFR-specific antibody therapy in CRC as well $(8,9)$. Thus, in a series of 113 patients receiving cetuximab or panitumumab, none of the 11 patients with tumors carrying BRAF mutations in a wild-type KRAS background responded to therapy. Accordingly, in two other studies, comprising a total of 142 patients with metastatic CRCs, cetuximab failed to exhibit any significant effect in patients with tumors displaying PIK3CA mutations in the background of wild-type KRAS (6). Therefore, we analyzed BRAF and PIK3CA genes in relation to CUP in this study (10).

Considering that in two of the present cases in which chemotherapy was given, the survival time from the initiation of treatment was 11 and 13 months, respectively, it is expected that the combined use of molecular target drugs will further extend prognoses in such cases (11), and application of molecular target drugs to cases in which resection is performed is also expected in the future. In CUP, it may be difficult to expect a complete cure from surgery, and surgery may be limited in its applicability. In the author's own treatment experience, it is difficult to conclude that surgery contributed to prognosis.

In conclusion, the mutation rate in CUP for codon 12 or 13 of the KRAS gene, and for PIK3CA is lower than in CRC, while the same mutation rate for BRAF is almost the same in the two; this means that the EGFR antibody may be effective for CUP. In our study, several cases who were administered chemotherapies showed a relatively good prognosis, and better prognosis will be expected by treatment with an EGFR antibody or a combination of an EGFR antibody and chemotherapy.

\section{References}

1. Hainsworth JD and Greco FA: Treatment of patients with cancer of an unknown primary site. N Engl J Med 329: 257-263, 1993.

2. Van Cutsem E, Köhne CH, Láng I, et al: Cetuximab plus irinotecan, fluorouracil, and leucovorin as first-line treatment for metastatic colorectal cancer: updated analysis of overall survival according to tumor KRAS and BRAF mutation status. J Clin Oncol 29: 2011-2019, 2011.

3. Moroni M, Veronese S, Benvenuti S, et al: Gene copy number for epidermal growth factor receptor (EGFR) and clinical response to anti EGFR treatment in colorectal cancer: a cohort study. Lancet Oncol 6: 279-286, 2006.

4. Hemminki K, Liu H, Hemminki A, et al: Power and limits of modern cancer diagnostics: cancer of unknown primary. Ann Oncol: Aug, 2011 (E-pub ahead of print).

5. Greco FA, Oien K, Erlander M, et al: Cancer of unknown primary: progress in the search for improved and rapid diagnosis leading toward superior patient outcomes. Ann Oncol: June, 2011 (E-pub ahead of print).

6. Baldus SE, Schaefer KL, Engers R, et al: Prevalence and heterogeneity of KRAS, BRAF, and PIK3CA mutations in primary colorectal adenocarcinomas and their corresponding metastases. Clin Cancer Res 16: 790-799, 2010.

7. Jonker DJ, O'Callaghan CJ, Karapetis CS, et al: Cetuximab for treatment of colorectal cancer. N Engl J Med 357: 2040-2048, 2007.

8. Di Nicolantonio F, Martini M, Molinari F, et al: Wild-type $\mathrm{BRAF}$ is required for response to panitumumab or cetuximab in metastatic colorectal cancer. J Clin Oncol 26: 5705-5712, 2008.

9. Sartore-Bianchi A, Martini M, Molinari F, et al: PIK3CA mutations in colorectal cancer are associated with clinical resistance to EGFR-targeted monoclonal antibodies. Cancer Res 69: 1851-1857, 2009.

10. Spindler KL, Pallisgaard N, Lindebjerg J, et al: EGFR related mutational status and association to clinical outcome of thirdline cetuximab-irinotecan in metastatic colorectal cancer. BMC Cancer 11: 107-115, 2011.

11. García-Foncillas J and Díaz-Rubio E: Progress in metastatic colorectal cancer: growing role of cetuximab to optimize clinical outcome. Clin Transl Oncol 12: 533-542, 2010. 